\title{
Leucine-Rich Repeat Kinase 2 Colocalizes with $\alpha$-Synuclein in Parkinson's Disease, but Not Tau-Containing Deposits in Tauopathies
}

\author{
George Perry a, biongwei Zhu ${ }^{a} \quad$ Asim K. Babar ${ }^{a} \quad$ Sandra L. Siedlak ${ }^{a}$ \\ Qiwei Yang $^{a}$ Genta Ito $^{c}$ Takeshi Iwatsubo $^{c}$ Mark A. Smith ${ }^{a}$ Shu G. Chen ${ }^{a}$ \\ ${ }^{a}$ Department of Pathology, Case Western Reserve University, Cleveland, Ohio, and ${ }^{\mathrm{b}}$ College of Sciences, \\ The University of Texas at San Antonio, San Antonio, Tex., USA; ' Department of Neuropathology and \\ Neuroscience, University of Tokyo, Tokyo, Japan
}

\section{Key Words}

Lewy bodies $\cdot$ Leucine-rich repeat kinase 2 - Parkinson's

disease $\cdot$ Synuclein and pathological conditions will deepen our understanding of its functions and molecular pathways relevant to the progression of Parkinson's disease and related disorders.

Copyright $\odot 2008$ S. Karger AG, Basel

\begin{abstract}
Background: Mutations in leucine-rich repeat kinase 2 (LRRK2) are thus far the most frequent genetic cause associated with autosomal dominant and idiopathic Parkinson's disease. Objective: To examine whether LRRK2 is directly associated with the pathological structures of Parkinson's disease, dementia with Lewy bodies, and other related disorders using highly specific antibodies to LRRK2. Results: LRRK2 antibodies strongly labeled brainstem and cortical Lewy bodies, the pathological hallmarks of Parkinson's disease and dementia with Lewy bodies, respectively. We found that $20-100 \%$ (mean $60 \%$ ) of $\alpha$-synuclein-positive Lewy bodies contained LRRK2. While antibodies raised against various regions of LRRK2 were previously shown to label recombinant LRRK2 on Western blots, only antibodies raised against the $\mathrm{N}$ - and $\mathrm{C}$-termini, but not the regions containing folded protein domains of LRRK2, immunolabeled Lewy bodies. In Alzheimer's disease, Hirano bodies were found to contain LRRK2 and the neurofibrillary tangles in progressive supranuclear palsy remained unlabeled. Conclusions: Information on the cellular localization of LRRK2 under normal
\end{abstract}

\section{Introduction}

Approximately $1-2 \%$ of individuals aged 65 years and older are affected by Parkinson's disease (PD), a condition characterized by selective loss of dopaminergic neurons and the presence of Lewy bodies (LBs), the hallmark neuropathology of the disease, in the substantia nigra. Mutations in genes encoding parkin, PINK1, and DJ-1 are correlated with autosomal recessive, early-onset familial PD, whereas genes encoding $\alpha$-synuclein and leucine-rich repeat kinase 2 (LRRK2) are linked to autosomal dominant PD. LRRK2 is a risk factor for both familial and sporadic PD, with the pathogenic G2019S mutation accounting for up to $6 \%$ of familial and $2 \%$ of sporadic cases [1]. Very recently, we have demonstrated that LRRK2, in addition to $\alpha$-synuclein and parkin [2], is the third component of LBs [3, 4]. In this study, we analyzed the spatial relationship between LRRK2 and $\alpha$-synuclein as well as tau in PD and other neurodegenerative diseases.

\section{KARGER}

Fax +4161306 1234

E-Mail karger@karger.ch

www.karger.com (c) 2008 S. Karger AG, Basel

Accessible online at:

www.karger.com/ndd
George Perry, $\mathrm{PhD}$

College of Sciences, University of Texas at San Antonio

One USTA Circle

San Antonio, TX 78249 (USA)

Tel. +1 210458 4450, Fax +1 210458 4445, E-Mail george.perry@utsa.edu 

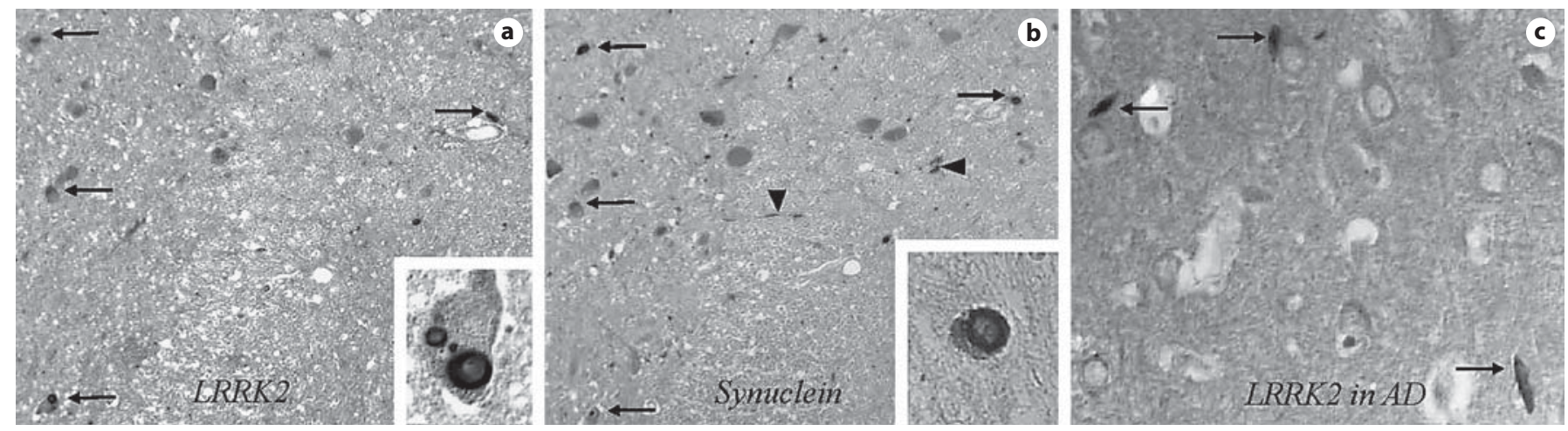

Fig. 1. In adjacent sections of PD brainstem, the same LBs often contain both LRRK2 (a) and $\alpha$-synuclein (b, arrows). Insets show a higher magnification of representative LBs. In AD hippocampus, Hirano bodies (arrows) are also found to contain LRRK2 (c).

\section{Materials and Methods}

Brain samples were obtained from patients with confirmed PD ( $\mathrm{n}=5$; aged 53-76 years), dementia with Lewy bodies (DLB; $\mathrm{n}=6$; aged 68-85 years), Alzheimer's disease ( $\mathrm{AD} ; \mathrm{n}=4$; aged 69-82 years), progressive supranuclear palsy (PSP; $n=2$; aged 76 and 83 years) and from control subjects ( $\mathrm{n}=4$; aged $42-79$ years). Tissues were fixed in formalin, embedded in paraffin and immunostained using 4 different highly specific antibodies against LRRK2 as previously described [3].

\section{Results}

Using antisera to 4 different regions of the LRRK2 protein, all of which could recognize LRRK2 from normal and PD brain homogenates by Western blot [3], only 2 with epitopes outside the 5 folded domains consistently and specifically labeled LBs in all PD cases studied [4]. Similar to $\alpha$-synuclein, both rim and core regions of brainstem LBs were detected by anti-LRRK2 (fig. 1). LBs stained for LRRK2 and $\alpha$-synuclein on adjacent sections revealed significant overlap (fig. 1a, b and insets). Quantification of the total number of LBs immunostained in sections of the brainstem from 5 cases of PD showed that LRRK2-positive LBs stained 20-100\% (mean 60\%) of those positive for $\alpha$-synuclein in adjacent sections. Small neuritic processes containing $\alpha$-synuclein were rarely labeled by anti-LRRK2 (fig. 1b, arrowheads). In cortical regions of $\mathrm{AD}$ and $2 \mathrm{DLB}$ cases, occasional morphologically characteristic Hirano bodies and neuronal cytoplasm were positive for LRRK2 (fig. 1c), but never neurofibrillary tangles nor amyloid deposits (not shown). In PSP, tangles, while intensely immunolabeled for tau, were negative for LRRK2 (not shown). In control brains, weak and uniform staining of neuronal cytoplasm was observed [3].

\section{Discussion}

Using a battery of specific antisera, we have demonstrated LRRK2 staining of LBs in the brainstem in idiopathic PD and in the cortex in DLB with 2 antibodies detecting the sequence outside the 5 folded domains, indicating that LRRK2 is indeed a component of LBs in these 2 diseases [3, 4]. Initially, a negative finding was reported using an antibody detecting the sequence within the folded LRR domain [5]; however, our positive findings $[3,4]$ were subsequently confirmed by others $[6,7]$. Here, we demonstrate a significant overlap of $\alpha$-synuclein-positive LBs with LRRK2. This is unlikely due to nonspecific cross-reactivity of the antibodies since the specificity of these two antibodies was demonstrated in our studies and also confirmed by other groups $[3,4,6,8]$. The differential detection of antibodies against different regions in the same protein is probably due to the inherent protein structure which results in the exposure or masking of specific epitopes, particularly for large proteins like LRRK2.

LRRK2 is predicted to contain several functionally important domains including mitogen-activated protein kinase and recent reports have confirmed that LRRK2 functions as a kinase and pathogenic mutations augment its activity in cell models [9]. Since the in vitro phosphorylation of $\alpha$-synuclein promotes its aggregation [10], the colocalization of the protein kinase LRRK2 and $\alpha$-sy- 
nuclein in the same LBs may provide a molecular mechanism associated with parkinsonism, including aberrant protein phosphorylation and subsequent aggregation. While we find no colocalization of LRRK2 with tau protein in AD and PSP cases prepared identically to the LRRK2-positive PD cases with all 4 antibodies used in these studies, another group using different fixation protocols [7] found LRRK2 in neurofibrillary tangles in AD with 1 of these antibodies. It is unclear what causes the difference between our observations.

Of interest is the presence of LRRK2 in Hirano bodies in both AD and DLB cases. Since Hirano bodies contain
F-actin and LRRK2 contains the Roc/GTPase domain, LRRK2 involvement in the regulation of cytoskeletal structures merits further investigation.

\section{Acknowledgements}

This project was supported in part by Alzheimer's Association, National Institute on Aging, National Parkinson Foundation, Johnson \& Johnson Corporate Office of Science and Technology, Philip Morris USA Inc., and Philip Morris International.

\section{References}

- 1 Hardy J, Cai H, Cookson MR, Gwinn-Hardy K, Singleton A: Genetics of Parkinson's disease and parkinsonism. Ann Neurol 2006; 60:389-398.

-2 Spillantini MG, Schmidt ML, Lee VM, Trojanowski JQ, Jakes R, Goedert M: Alphasynuclein in Lewy bodies. Nature 1997;388: 839-840.

-3 Zhu X, Babar A, Siedlak SL, Yang Q, Ito G, Iwatsubo T, Smith MA, Perry G, Chen SG: LRRK2 in Parkinson's disease and dementia with Lewy bodies. Mol Neurodegener 2006; 1:17.

4 Zhu X, Siedlak SL, Smith MA, Perry G, Chen SG: LRRK2 protein is a component of Lewy bodies. Ann Neurol 2006;60:617-618; author reply 618-619.
5 Giasson BI, Covy JP, Bonini NM, Hurtig HI, Farrer MJ, Trojanowski JQ, Van Deerlin VM: Biochemical and pathological characterization of Lrrk2. Ann Neurol 2006;59:315-322.

6 Greggio E, Jain S, Kingsbury A, Bandopadhyay R, Lewis P, Kaganovich A, van der Brug MP, Beilina A, Blackinton J, Thomas KJ, Ahmad R, Miller DW, Kesavapany S, Singleton A, Lees A, Harvey RJ, Harvey K, Cookson MR: Kinase activity is required for the toxic effects of mutant LRRK2/dardarin. Neurobiol Dis 2006;23:329-341.

7 Miklossy J, Arai T, Guo JP, Klegeris A, Yu S, McGeer EG, McGeer PL: LRRK2 expression in normal and pathologic human brain and in human cell lines. J Neuropathol Exp Neurol 2006;65:953-963.
8 Hatano T, Kubo SI, Imai S, Maeda M, Ishikawa K, Mizuno Y, Hattori N: Leucine-rich repeat kinase 2 associates with lipid rafts. Hum Mol Genet 2007;16:678-690.

-9 West AB, Moore DJ, Biskup S, Bugayenko A, Smith WW, Ross CA, Dawson VL, Dawson TM: Parkinson's disease-associated mutations in leucine-rich repeat kinase 2 augment kinase activity. Proc Natl Acad Sci USA 2005; 102:16842-16847.

10 Iwatsubo T: Aggregation of alpha-synuclein in the pathogenesis of Parkinson's disease. J Neurol 2003;250(suppl 3):III11-III14. 